\title{
Compromised Network Design Model for the Strategic Alliance of Service Centers and Consolidation Terminals in Express Courier Services
}

\author{
Ki Ho Chung ${ }^{1} \cdot$ Hyun Jeung $\mathrm{Ko}^{2} \cdot$ Chang Seong $\mathrm{Ko}^{3^{\dagger}}$ \\ ${ }^{1}$ Department of Management Information Systems, Kyungsung University, Busan, 608-736, Korea \\ ${ }^{2}$ Department of Logistics, Kunsan National University, Kunsan, 573-701, Korea \\ ${ }^{3}$ Department of Industrial and Management Engineering, Kyungsung University, 608-736, Korea

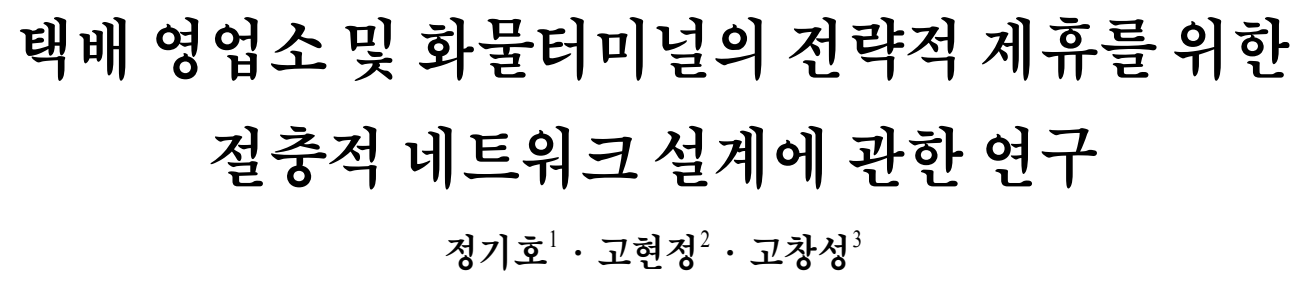 \\ ${ }^{1}$ 경성대학교 경영정보학과 $/{ }^{2}$ 군산대학교 물류학과 $/{ }^{3}$ 경성대학교 산업경영공학과
}

\begin{abstract}
The express courier business in Korea has been recognized as one of promising business sectors with the annual growth rate of over 10 percent since 2001. As such, many domestic and foreign companies have been established and are fiercely competing to extend their own market share. The severe competition has forced them to focus on achieving cost competitiveness and high level of customer service. Small and medium-sized enterprises(SMEs) with relatively poor network infrastructure, in particular, are facing tougher challenges to cost reduction and efficiency improvement in their logistics networks. Strategic alliances among SMEs may be an effective way to cope with these challenges. Therefore, this study proposes an integer programming model for compromised network design and its solution procedure based on maxmin and maxsum criteria for strategic alliances. The applicability and efficiency of the proposed model are demonstrated through an illustrative numerical example.
\end{abstract}

Keywords: Express Courier Service, Compromised Network Design, Strategic Alliance, Service Center, Consolidation Terminal

\section{Introduction}

Courier service is nowadays recognized as one of the most efficient delivery methods due to the increasingly far-flung nature of business operations in the global economy with the popularity of e-commerce, TV home shopping, telemarketing, and door-to-door deliveries. In particular, the total Korean market size of courier service industry is expected to reach up to a four trillion won by 2014 with the annual growth rate of over 10 percent since 2001. As such, the courier business

This research was supported by Basic Science Research Program through the National Research Foundation of Korea(NRF) funded by the Ministry of Education, Science Technology (2010-0027995).

$\uparrow$ Corresponding author : Professor Chang Seong Ko, Department of Industrial and Management Engineering, Kyunsung University, Busan, 608-736, Korea, Fax :+82-51-621-2454, E-mail : csko@ks.ac.kr

Received September 7, 2011; Revision Received October 18, 2011; Accepted November 10, 2011. 
held great potential for successful business venture in Korea, and many Korean companies providing domestic courier services have been established. Moreover, a number of global foreign companies have also entered into Korean market. These domestic and international companies are fiercely competing to extend their own market share, and the severe competition has forced them to focus on achieving cost competitiveness as well as providing a high level of customer service. Under the current business environment of courier service industry, small and medium enterprises (SMEs) with relatively poor network infrastructure, in particular, are facing tougher challenges to cost reduction and efficiency improvement in their logistics networks. To cope with the challenges, the adoption of strategic alliance (e.g., sharing network infrastructure such as service centers and consolidation terminals) may be an effective way of maintaining their competitiveness. The strategic alliance among SMEs can create economies of scale leading to cost reduction of operation. Through an efficient operation of sharing service centers, for example, all the allied companies may realize an increase in net profits. They may also provide better services to customers by cooperatively sharing their existing facilities. Consequently, SMEs may efficiently compete for expanding their market share without further investment.

There have been a few studies on various types of strategic alliances in logistics. Chopra and Meindl (2004), Min (1996), and Simchi-Levi et al. (2003) pointed out that a significant amount of cost savings may be realized by consolidating two or more facilities as a type of strategic alliances in supply chain management. The cost efficiencies result from reduced space by increasing storage density, reduced safety stock inventory by consolidating stock, more efficient methods and equipment to handle an increased volume, and reduced overhead and redundant management costs. In transportation operation, the cost savings could also be achieved by taking advantage of consolidated shipment to its customers rather than multiple shipments. Cachon and Lariviere (1999) proposed a methodology for optimal capacity allocation. There have also been many studies directly related to the design of courier service network. The study conducted by Cheung et al. (2001) was the first to examine a service network design problem encountering express couriers such as DHL Hong Kong. They proposed a hybrid optimization/simulation model that aimed to maximize service coverage and service reliability by adjusting cutoff time. Ko et al. (2010) suggested an approach to the reconfiguration of an express courier service network with respect to assignments of service centers to consolidation terminals and adjustments of their cutoff times. They proposed an integer programming model and a genetic algorithm-based solution procedure for allowing express couriers to maximize their incremental profit. Recently, Chung et al. (2009) proposed a network design model for strategic alliances among express courier service companies by monopoly of service centers. Chung et al. (2010) extended their previous study to the problem of sharing consolidation terminals of individual companies. An integer programming model and its solution procedure based on a fuzzy set theoretic approach were developed. However, the prior studies dealing with a network design model with respect to strategic alliance have been performed under the assumption that the consolidation terminals could be only shared by the service centers selected as candidates for alliance in merging regions.

Apart from the previous studies, this study especially takes into account the capacity share of a consolidation terminal among allied companies for the shipments of service centers selected in not only merging regions but also non-merging regions. The problem situation thus comes to be more realistic and becomes more complex to solve. Therefore, this study proposes a compromised network design model to facilitate strategic alliances among SMEs in courier service industry. The objective of the proposed model is how to increase the net profit of each allied company by harnessing its under-utilized service centers due to low demand and sharing consolidation terminals with available processing capacities for the open service centers in merging and non-merging regions. A multi- objective integer programming model and its solution procedure based on maxmin and maxsum criteria are developed and then a numerical example is carried out to verify the applicability of the proposed model to real world problems.

\section{Problem Definition}

A courier service network generally consists of customer zones, service centers, and consolidation terminals. In the service center, express courier companies receive shipment request from customer zones, pick up parcels, screen, sort, document, label, and consolidate them before transshipping them in bulk to a consolidation terminal. In this way, the service center acts as a temporary storage facility connecting customers to a consolidation terminal. At the consolidation terminal, the packages delivered from service centers are combined, screened, sorted, and then loaded onto delivery trucks for their destinations. Thus, the productivity and service level of an express courier service are highly related to the efficient management of 
service centers and consolidation terminals while maintaining an appropriate level of customer satisfaction. For the purpose of this study, a network design model with strategic alliance may be classified into one of the following four models :

- Model (a) is a service center level collaboration model. Each participating company only collaborates in pick-up/delivery at consolidated service centers while its own terminals are exclusively operated. That is, a consolidated service center is not reallocated to each company. The objective of this model is to minimize the operation cost of service centers by reducing the number of service centers in a region with a low demand.

- Model (b) is a terminal level collaboration model. The participating companies may share other company's terminals and reallocate service centers to the terminals. The goal of this model is to improve the utilization rate of existing terminals of participating companies.

- Model (c) is another form of terminal level collaboration. This is a location/allocation model which simultaneously determines whether the existing terminals are still open or not and how to reallocate service centers to the open terminals. Its objective is to minimize the total operation cost by reducing the number of terminals and reassigning the service centers to the terminals.

- Model (d) is an extended version of terminal level collaboration model which can be applied when participating companies have consolidated through merger and acquisition (M\&A). This model determines whether the existing terminals are open or not with the consideration of changing terminal capacities. The goal of this model is to minimize the total operation cost and to maximize the utilization rate of terminals and service centers.

The strategic alliance model investigated in this paper is a hybrid model of the Models (a) and (b) discussed above. In this study, companies participating in the alliance collaborate in pick-up/delivery together at the merged service centers and share the capacity of consolidation terminals by reassigning all the open service centers to the consolidation terminals. We assume that there are several regions in which each participating company has a service center with low demand of daily pick-up, so-called merging regions and the others are called as non-merging regions. Service centers and consolidation terminals are also assumed to be operated according to the following principles:

- Within a merging region after alliance, only one service center stays open while remaining service centers are closed.

- The open service center after alliance is also responsible for pick-up and delivery of all the amo- unts of other company's service centers within the same merging region.

- The current existing consolidation terminals are still used even after strategic alliance.

- Each company's service centers are divided into two types; Service centers in a merging region are defined to be Type I service centers, and Type II service centers do not belong to any merging regions.

- Both the open Type I service centers in each merging region and Type II service centers may be reallocated to all the company's consolidation terminal while satisfying the processing capacity of terminal.

The selection of open service center in each merging region and the reassignment of open Type I and Type II service centers to the consolidation terminals are formulated as an integer programming model and the solution procedure will also be developed.

\section{Mathematical Model}

This section describes the integer programming model to maximize the expected profit increase of each participating company. Suppose that there are $n$ express courier companies, and that the location of terminals and service centers managed by each company are given. The underlying assumption is that there are $m$ regions in which all $n$ companies possess one service center independently with relatively small amount of shipment. Under the condition that the daily pick-up amount for the service centers in each region and the processing capacity of terminals per day are given, the problem is to maximize the profit of each company by selecting one among $n$ service centers within each region and reallocating the open Type I and Type II service centers to terminals subject to the processing capacity of all the terminals. It is assumed that all terminals operated by one company are also available to other companies after alliance. The following notations are introduced for developing the mathematical model for the problem :

$I$ : Set of service regions in which service centers are to be merged, $I=\{1,2, \cdots, m\}$

$J$ : Set of express courier companies, $J=\{1,2, \cdots, n\}$

$S_{j}$ : Set of Type II service centers of company $j, j \in J$

$T_{j}$ : Set of terminals for company $j, j \in J$

$T: T_{1}+T_{2}+\cdots+T_{n}$

$d_{i j}^{1}$ : Daily pick-up amount of company $j$ 's Type I service center in region $i, i \in I, j \in J$

$D_{i}$ : Sum of daily pick-up amount of all the service centers within region $i, i \in I$, i.e., $D_{i}=\sum_{j \in J} d_{i j}$ 
$d_{j l}^{2}$ : Daily pick-up amount of company $j$ 's Type II service center $l, j \in J, l \in S_{j}$

$a_{i j k}$ : Indicator variable such that $a_{i j k}=1$, if daily pick-up amount of company $j$ 's

Type I service center in region $i$ is assigned to terminal $k$ of company $j$

before alliance, $a_{i j k}=0$, otherwise, $i \in I, j \in J, k \in T_{j}$

$b_{j l k}$ : Indicator variable such that $b_{j l k}=1$, if daily pick-up amount of company $j$ 's

Type II service center $l$ is assigned to terminal $k$ of company $j$ before alliance,

$b_{j l k}=0$, otherwise, $j \in J, l \in S_{j}, k \in T_{j}$

$Q_{j k}$ : Capacity for terminal $k$ of company $j$, $j \in J, k \in T_{j}$

$r_{i j}$ : Net profit contributed by one unit of pick-up amount for company $j$ 's Type I service center in region $i, i \in I, j \in J$

$p_{j k}$ : Net profit obtained by terminal $k$ when one unit of pick-up amount in a merging region is assigned to terminal $k$ of company $j, j \in J, k \in T_{j}$

$q_{j l k}$ : Net profit obtained by terminal $k$ when one unit of pick-up amount of company $j$ 's

Type II service center $l$ is assigned to terminal $k$, $j \in J, l \in S_{j}, k \in T$

$f_{i j}$ : Daily fixed cost accruing from operating company $j$ 's Type I service center in region $i$, $i \in I, j \in J$

$x_{i j}$ : Binary variable such that $x_{i j}=1$, if company $j$ 's Type I service center in region $i$ is still open, $x_{i j}=0$ otherwise, $i \in I, j \in J$

$y_{i j k}$ : Binary variable such that $y_{i j k}=0$, if the sum of pick-up amount of all the Type I service centers in region $i$ is assigned to terminal $k$ of company $j, y_{i j k}=0$, otherwise, $i \in I, j \in J, k \in T_{j}$

$z_{j l k}$ : Binary variable such that $z_{j l k}=1$, if all the pick-up amount of company $j$ 's Type II service center $l$ is assigned to terminal $k, z_{j k}=0$, otherwise, $j \in J, l \in S_{j}, k \in T$

When Type I service centers are merged and reallocated to a terminal, changes in net profit and reduction of operating cost for each company are calculated as follows: Consider a company called $j^{\prime}$. If the Type I service center of company $j^{\prime}$ is still open in region $i$, then the profit of company $j^{\prime}$ resulting from merging Type I service centers increases by $r_{i j^{\prime}}\left(D_{i}-d_{i j^{j}}\right)$. On the other hand, if Type I service center of company $j^{\prime}$ is close in region $i$, then the profit of company $j^{\prime}$ decreases by $-r_{i j} d_{i j}$. Thus the net profit of company $j^{\prime}$ by merging Type I service centers for all merging regions can be written as $\sum_{i \in I} r_{i j^{\prime}}\left(D_{i} x_{i j^{\prime}}-d_{i j^{\prime}}\right)$. If the service center of company $j^{\prime}$ is open in region $i$ and the sum of daily pick-up amount of all the service centers in the region is assigned to another company's terminal, then the net profit of company $j^{\prime}$ decreases because the profit from processing the amount in the terminal of company $j^{\prime}$ will be transferred to other company. On the other hand, if other company's Type I service center is merged in a region and it is assigned to a terminal of company $j^{\prime}$, the profit of company $j^{\prime}$ increases by $\sum_{i \in I k \in T_{j}} \sum_{j^{\prime} k}\left(y_{i j^{\prime} k}-a_{i j^{\prime} k} x_{i j^{\prime}}\right) D_{i}$. And the sum of daily fixed operating cost reduced by closing service centers of company $j^{\prime}$ can be written as $\sum_{i \in I} f_{i j^{\prime}}\left(1-x_{i j}\right)$. Next, the profit of company $j^{\prime}$ by reassigning Type II service centers to a terminal is $\sum_{j \in J i \in S_{j}} \sum_{k \in T_{j}} q_{j k} d_{j l}^{2} z_{j l k}-\sum_{l \in S_{j}} \sum_{k \in T_{j}} q_{j l k} d_{j l}^{2} b_{j l k}$. Then the objective function for company $j^{\prime}$ can be derived as follows :

$$
\begin{aligned}
& \operatorname{Max} Z_{j^{\prime}}(x)=\sum_{i \in I} r_{i j^{\prime}}\left(D_{i} x_{i j^{\prime}}-d_{i j^{\prime}}\right) \\
& +\sum_{i \in I} \sum_{k \in T_{j}} p_{j^{\prime} k}\left(y_{i j^{\prime} k}-a_{i j^{\prime} k} x_{i j^{\prime}}\right) D_{i}+\sum_{i \in I} f_{i j^{\prime}}\left(1-x_{i j^{\prime}}\right) \\
& +\sum_{j \in J} \sum_{l \in S_{i}} \sum_{k \in T_{j}} q_{j l k} d_{j l}^{2} z_{j l k}-\sum_{l \in S_{i}} \sum_{k \in T_{j}} q_{j^{\prime} l k} d_{j^{\prime} l}^{2} b_{j^{\prime} k} \\
& =\sum_{i \in I}\left(r_{i j} D_{i}-f_{i j^{\prime}}-\sum_{k \in T_{j}} p_{j^{\prime} k} a_{i j^{\prime} k} D_{i}\right) x_{i j^{\prime}} \\
& +\sum_{i \in I} \sum_{k \in T_{j}^{j}} p_{j^{\prime} k} D_{i} y_{i j k}+\sum_{j \in J} \sum_{l \in S_{j}} \sum_{k \in T_{j}} q_{j l k} d_{j l}^{2} z_{j l k} \\
& +\sum_{i \in I}\left(f_{i j^{\prime}}-r_{i j^{\prime}} d_{i j^{\prime}}\right)-\sum_{l \in S_{j}} \sum_{k \in T_{i}^{\prime}} q_{j^{\prime} k} d_{j^{\prime} l^{2}}^{2} b_{j^{\prime} l k}
\end{aligned}
$$

Since the last two terms $\sum_{i \in I}\left(f_{i j^{\prime}}-r_{i j^{\prime}} d_{i j^{\prime}}\right)-\sum_{l \in S_{j}^{\prime}} \sum_{k \in T_{j}} q_{j^{\prime} l k} d_{j^{\prime} l}^{2}$ $\sum_{i \in I} b_{j l k}$ are constant, they may be calculated and expressed as $C_{j}$ in the objective function. Thus, the problem can be described as the following multi-objective integer programming model $(\mathrm{P})$ with $n$ objective functions :

(p)

$$
\begin{aligned}
\operatorname{Max} Z_{1}(x)= & \sum_{i \in I}\left(r_{i 1} D_{i}-f_{i 1}-\sum_{k \in T_{1}} p_{1 k} a_{i 1 k} D_{i}\right) x_{i 1} \\
& +\sum_{i \in I k \in T_{1}} \sum_{1 k} D_{i} y_{i 1 k}+\sum_{j \in J \in S_{j} \in T_{1}} \sum_{j l k} d_{j l}^{2} z_{j l k}+C_{1} \\
& \vdots \\
\operatorname{Max} Z_{n}(x)= & \sum_{i \in I}\left(r_{i n} D_{i}-f_{i n}-\sum_{k \in T_{n}} p_{n k} a_{i n k} D_{i}\right) x_{i n} \\
& +\sum_{i \in I} \sum_{k \in T_{n}} p_{n k} D_{i} y_{i n k}+\sum_{j \in J l \in S_{j}} \sum_{k \in T_{n}} q_{j l k} d_{j l}^{2} z_{j l k}+C_{n}
\end{aligned}
$$

s.t. $\sum_{j \in J} x_{i j}=1$,

$i \in I$

$\sum_{j \in J} \sum_{k \in T_{j}} y_{i j k}=1$

$i \in I$

$\sum_{k \in T} z_{j l k}=1$,

$j \in J, l \in S_{j}$

$\sum_{i \in I} D_{i} y_{i j k}+\sum_{j \in J} \sum_{l \in S_{j}} d_{j^{\prime}}^{2} z_{j l k}^{\prime} \leq Q_{j k,} \quad j \in J, k \in T_{j}$

$x_{i j} \in\{0,1\}$,

$$
\begin{array}{r}
i \in I, \quad j \in J \\
i \in I, \quad j \in J, k \in T_{j} \\
j \in J, l \in S_{j}, k \in T
\end{array}
$$$$
y_{i j k} \in\{0,1\} \text {, }
$$$$
z_{j l k} \in\{0,1\} \text {, }
$$ 
The objective function (1) consists of $n$ conflicting objectives corresponding to $n$ companies. Each objective function represents the sum of net profit increases through merging Type I service centers and net profit increases by allocating Type II service centers to terminals. Constraint (2) assures that only one service center is open in a region and all the others closed. Constraint (3) implies that the entire pick-up amount consolidated in a region should be assigned to only one terminal. Constraint (4) implies that every Type II service center must be allocated to only one terminal. Constraint (5) shows that the sum of daily pick-up amount of Type I and Type II service centers allocated to the terminal cannot exceed the processing capacity of the terminal. Constraints $(6) \sim(8)$ represent binary variables.

\section{Solution Procedure}

Since there are $n$ objective functions in model (P) which represent the net profit increases of $n$ companies, there exists a trade-off relationship among them. This paper proposes two methods using maxmin and maxsum criteria. Applying a maxmin criterion, the minimum of participating companies' profits is denoted by a, i.e.,

$$
\alpha=\operatorname{Min}\left\{Z_{1}(x), Z_{2}(x), \cdots, Z_{n}(x)\right\}
$$

Then, the problem can be written as follows :

$$
\begin{array}{ll}
\text { Max } & \alpha \\
\text { s.t. } & Z_{i}(x) \geq \alpha, \quad i=1, \cdots, n \\
& x \in F(x),
\end{array}
$$

where $F(x)$ is the solution set satisfying constraints (2) $\sim(8)$ of problem (P).

Next, a maxsum criterion is to maximize the sum of the net profits realized by all the companies within strategic alliance. The problem based on the maxsum criterion may be formulated as (P2).

$$
\begin{aligned}
\operatorname{Max} Z= & \sum_{i \in I j^{\prime} \in J} \sum_{j}\left(r_{i j^{\prime}} D_{i}-f_{i j^{\prime}}-\sum_{k \in T_{j}} p_{j k} a_{i j^{\prime} k} D_{i}\right) x_{i j^{\prime}} \\
& +\sum_{i \in I j^{\prime} \in J k} \sum_{J_{k} \in T_{j}} p_{j^{\prime} k} D_{i} y_{i j^{\prime} k}+\sum_{j^{\prime} \in J j \in J \in S_{j} k \in T_{j}} \sum_{j k} \sum_{j l} d_{j l^{2}}^{2} z_{j l k} \\
\text { s.t. } x \in & F(x)
\end{aligned}
$$

The objective function of (P2) is expressed as the sum of $n$ objective functions in (P), which is the sum of the net profits realized by $n$ companies. It is intuitive that the total profit increases with the maxsum criterion is greater than the total profit with the maxmin criterion. However, it may be desirable to distribute the realized total profit evenly among all the companies.

\section{Numerical Example}

An example problem involving two express courier companies is described below to demonstrate the proposed approach in this study. For the purpose of simplicity, only pick-up amount is included in the model and delivery amount is not considered. The total number of regions for merging service centers is fixed to five, and the number of terminals owned by each company to two. The set of terminals for company 1 is $T 1$ $=\{1,2\}$ and the set of terminals for company 2 is $T 2=$ $\{3,4\}$. Every service center of company 1 is allocated to terminal 1 or 2 and every service center of company 2 is allocated to terminal 3 or 4 by means of random-number generation. The daily pick-up amount for each Type I service center is determined in the range between 10 and 50 units and the daily pick-up amount of Type II service center is assigned between 100 and 500 units through random-number generation. In addition, service center closedown results in the reduction of daily fixed cost for maintaining and operating the service centers, and these cost reductions should be converted to net present value. These are obtained by generating random-number between $\$ 50$ and $\$ 100$ per day. The terminal capacity is equally assigned to 2,000 units for every terminal of two companies. <Table 1> displays the amount of daily pick-up, allocated terminal, and the daily fixed cost of Type I service center for two companies. The daily pick-up amounts of Type II service centers are also shown in $<$ Table $2>$.

Table 1. Data for Type I service centers

\begin{tabular}{c|c|c|c|c|c|c}
\hline \multirow{2}{*}{$\begin{array}{c}\text { Merging } \\
\text { Region }\end{array}$} & \multicolumn{2}{|c|}{$\begin{array}{c}\text { Pick-Up } \\
\text { Amount }\end{array}$} & \multicolumn{2}{c|}{$\begin{array}{c}\text { Allocated } \\
\text { Terminal }\end{array}$} & \multicolumn{2}{c}{$\begin{array}{c}\text { Daily Fixed } \\
\text { Cost }\end{array}$} \\
\cline { 2 - 7 } & $\mathrm{C} 1$ & $\mathrm{C} 2$ & $\mathrm{C} 1$ & $\mathrm{C} 2$ & $\mathrm{C} 1$ & $\mathrm{C} 2$ \\
\hline 1 & 40 & 13 & 1 & 3 & 82 & 57 \\
\hline 2 & 50 & 37 & 1 & 3 & 58 & 91 \\
\hline 3 & 35 & 15 & 1 & 4 & 64 & 51 \\
\hline 4 & 37 & 42 & 2 & 4 & 87 & 64 \\
\hline 5 & 34 & 10 & 2 & 3 & 71 & 82 \\
\hline
\end{tabular}


Table 2. Data for Type II service centers

\begin{tabular}{c|c|c}
\hline Non-Merging Region & Company 1 & Company 2 \\
\hline 1 & 483 & 447 \\
\hline 2 & 478 & 160 \\
\hline 3 & 384 & 192 \\
\hline 4 & 354 & 219 \\
\hline 5 & 107 & 278 \\
\hline 6 & 382 & 127 \\
\hline 7 & 114 & 298 \\
\hline 8 & 257 & 363 \\
\hline 9 & 357 & 364 \\
\hline 10 & 201 & 267 \\
\hline
\end{tabular}

Table 3. Optimal solution and allocation of the Type I service centers to terminals

\begin{tabular}{c|c|c}
\hline \multirow{2}{*}{$\begin{array}{c}\text { Merging } \\
\text { Region }\end{array}$} & \multicolumn{2}{|c}{ Open/Assigned Terminal } \\
\cline { 2 - 3 } & Company 1 & Company 2 \\
\hline 1 & 0 & $1 / 1$ \\
\hline 2 & $1 / 1$ & 0 \\
\hline 3 & 0 & $1 / 4$ \\
\hline 4 & 0 & $1 / 3$ \\
\hline 5 & $1 / 4$ & 0 \\
\hline
\end{tabular}

Table 4. Optimal allocation of the Type II service centers to terminals

\begin{tabular}{c|c|c}
\hline Non-Merging Region & Company 1 & Company 2 \\
\hline 1 & 1 & 4 \\
\hline 2 & 2 & 3 \\
\hline 3 & 1 & 1 \\
\hline 4 & 3 & 2 \\
\hline 5 & 1 & 4 \\
\hline 6 & 1 & 4 \\
\hline 7 & 4 & 2 \\
\hline 8 & 3 & 2 \\
\hline 9 & 3 & 4 \\
\hline 10 & 4 & 3 \\
\hline
\end{tabular}

The optimal solution for maxmin criterion can be obtained using Excel Solver. <Table 3> shows the optimal solution. Company 1's service centers are selected to be open in regions 2 and 5 , while the service centers of company 2 are open in other regions. The optimal allocation of open Type I and Type II service centers to terminals is shown in $<$ Table $3>$ and $<$ Table $4>$. The values of objective functions for companies 1 and 2 are $Z 1=3,119, Z 2=3,119$, respectively. The profits realized with maxsum criteria are 3,517 and 2,722 for companies 1 and 2, respectively. The problem has also been solved with the objective function maximizing each company's own profit to compare the performance of the compromised solutions. It can be observed from Table 5 that the compromised solutions based on maxmin and maxsum criterion provide a better balance in each company's profit.

Table 5. Comparisons between maxmin and maxsum criteria

\begin{tabular}{c|c|c|c}
\hline \multirow{2}{*}{ Criterion } & \multicolumn{2}{|c|}{ Objective Function Value } & \multirow{2}{*}{ Sum } \\
\cline { 2 - 3 } & Company 1 & Company 2 & \\
\hline Max $Z_{1}(x)$ & 3,643 & 2,548 & 6,191 \\
\hline Max $Z_{2}(x)$ & 2,605 & 3,600 & 6,205 \\
\hline Maxmin & 3,119 & 3,119 & 6,238 \\
\hline Maxsum & 3,517 & 2,722 & 6,239 \\
\hline
\end{tabular}

\section{Conclusions}

As the market size of express courier industry grows rapidly, competition among express courier companies is becoming more severe. Especially, small and medium-sized companies have relatively poor network infrastructure and are facing challenges to cost reduction and efficiency improvement in their logistics networks. To cope with the challenges, strategic alliance via sharing network infrastructure such as service centers and consolidation terminals is recommended. The adoption of strategic alliance may be an effective way of enhancing their competitiveness because it has some meaningful advantages. The strategic alliance among companies can create economies of scale leading to cost reduction of operation. Through an efficient sharing of service centers and/or consolidation terminals, all the allied companies may realize an increase in net profits. They may also provide better services to customers by cooperatively utilizing their existing facilities. Consequently, they may efficiently compete for expanding their market share without further investment. In this study, we present a network design model for strategic alliance among small and medium sized express courier service companies and develop a solution procedure. Other problems in strategic alliance, such as co-operation of terminals, maintenance/closedown of existing terminals, and capacity change of terminals, are to be included in the future research. 


\section{References}

Cachon, G. P. and Lariviere, M. A. (1999), Capacity choice and allocation : strategic behavior and supply chain performance, Management Science, 45(8), 1091-1108.

Cheung, W., Leung, L. C., and Wong, Y. M. (2001), Strategic service network design for DHL Hong Kong, Interfaces, 31(4), 1-14.

Chopra, S. and Meindl, P. (2004), Supply chain management : strategy, planning and operation, NJ : Pearson Prentice Hall.

Chung, K. H., Ko, C. S., Hwang Y. M., and Rho, J. J. (2010), Network design for strategic alliance in express courier services : a fuzzy set approach, International Journal of Innovative Computing, Information and Control, 9(1), 349359.

Chung, K. H., Rho, J. J., and Ko, C. S. (2009), Strategic alliance model with regional monopoly of service centers in express courier services, International Journal of Service and Operation Management, 5(6), 774-786.

Cooper, M. C. (1983), Freight consolidation and warehouse location strategies in physical distribution systems, Journal of Business Logistics, 4(2), 53-74.

Ko, H. J., Lee, H. J., and Ko, C. S. (2010), A study on the design of express courier service network based on the cutoff time adjustments, International Journal of Innovative Computing, Information and Control, 9(1), 335-347.

Min, H. (1996), Consolidation terminal location-allocation and consolidated routing problems, Journal of Business Logistics, 17(2), 235-263.

Simchi-Levi, D., Kaminsky, P., and Simchi-Levi, E. (2003), Designing and managing the supply chain : concepts, strategies, and case studies, NY : McGraw-Hill. 